\title{
Jeder Vierte leidet ein Jahr später an Angina pectoris
}

Herzinfarktpatienten, die keine relevanten Koronarstenosen aufweisen, leiden nach dem Infarkt genauso häufig an Angina pectoris wie Patienten mit Koronarobstruktion.

— Bei rund zehn von 100 Herzinfarktpatienten sind keine klinisch relevanten Koronarstenosen festzustellen. Vermutlich wirken hier verschiedene Mechanismen wie Spasmen, Thrombusbildung und -lösung zusammen und lösen schließlich den Infarkt aus. Frühere Studien haben gezeigt, dass diese Patienten seltener weitere Infarkte erleiden. In der Sterblichkeit unterscheiden sie sich auf längere Sicht aber nicht von $\mathrm{Pa}$ tienten mit relevanten Stenosen.

Wenig ist darüber bekannt, wie es um die Lebensqualität von Infarktpatienten ohne Koronarstenosen bestellt ist. Speziell geht es um die Frage, wie häufig sie von Anginabeschwerden geplagt werden. Kardiologen der University of Missouri in Kansas City untersuchten 5.539 Patienten, die sich nach einem Infarkt einer Angiografie unterzogen.

Bei 6,9\% der Infarktpatienten fand sich kein angiografischer Nachweis einer relevanten Stenose. Sie hatten im Vergleich zu Patienten mit obstruktiver koronarer Herzkrankheit meist Nicht-ST-Hebungsinfarkte ( $87 \%$ vs. $51 \%$ ). Frauen waren in der Mehrzahl (57\% vs. $30 \%$ ). Infarktpatienten ohne Stenosen hatten vor dem Infarkt seltener eine Angina pectoris gehabt ( $43 \%$ vs. $48 \%$ ). Ein Jahr nach dem Infarkt waren sie aber häufiger betroffen ( $25 \%$ vs. $21 \%)$. Nach Abgleich von soziodemografischen und klinischen Faktoren ergab sich kein signifikanter Unterschied in der Anginainzidenz. Insgesamt ging es den Patienten ohne obstruktive KHK schlechter

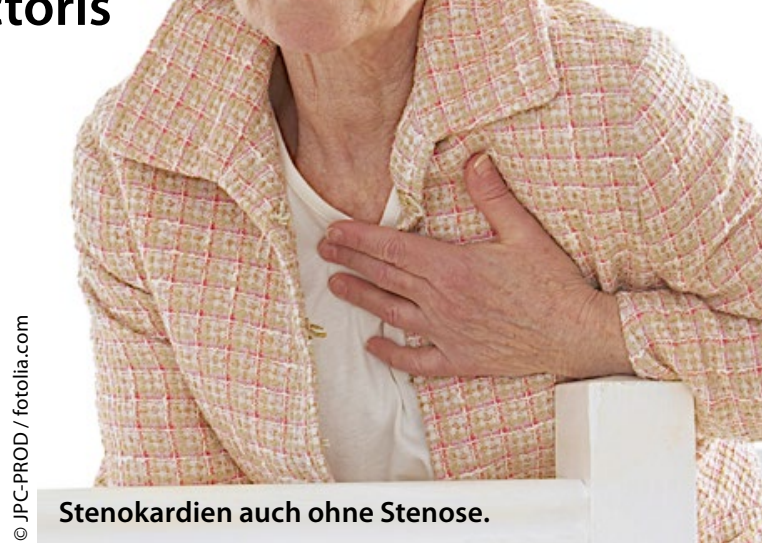

und sie waren unzufriedener mit ihrer Therapie. Sie bekamen seltener Betablocker und häufiger Kalziumantagonisten verschrieben. Herzinfarktpatienten ohne Koronarstenosen werden in der Sekundärprävention weniger aggressiv und seltener leitliniengerecht behandelt, so die Autoren.

$\mathrm{rb}$.

- Grodzinsky A et al. Eur Heart J Qual Care Clin Outcomes, online 20. August 2015; doi:10.1093/ehjqcco/qcv014

\section{Hier steht eine Anzeige.}

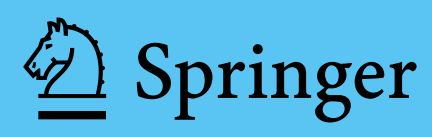

